\title{
Historia de incendios en un bosque de pino de la sierra de Manantlán, Jalisco, México
}

\author{
Fire history in a pine forest in Sierra de Manantlán, Jalisco, Mexico
}

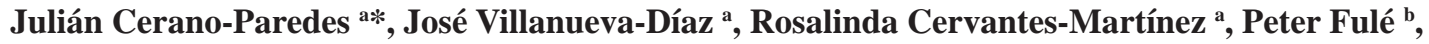 \\ Larissa Yocom ${ }^{\text {b }}$, Gerardo Esquivel-Arriaga a, Enrique Jardel-Peláez ${ }^{c}$ \\ *Autor de correspondencia: ${ }^{a}$ Centro Nacional de Investigación Disciplinaria en Relación Agua-Suelo-Planta-Atmósfera, \\ Km 6,5 margen derecha del Canal Sacramento, Gómez Palacio, Durango, México, código postal 35140, \\ tel.: (871) 1590104 y 05, cerano.julian@inifap.gob.mx \\ ${ }^{\mathrm{b}}$ Northern Arizona University, School of Forestry, P.O. Box 15018, Flagstaff, Arizona, USA. \\ c Universidad de Guadalajara, Instituto Manantlán de Ecología y Conservación de la Biodiversidad, \\ Centro Universitario de la Costa Sur, Jalisco, México.
}

\begin{abstract}
SUMMARY
Sierra de Manantlán Biosphere Reserve (RBSM) in Jalisco is the most important reserve in western Mexico, where fires are one of the main forest disturbances. In order to reconstruct historical fire regimes, partial sections of Pinus douglasiana with fire scars were collected. Using dendrochronological techniques, the exact dating of 293 scars from 51 trees allowed the reconstruction of fire frequency for the period 1867-2010. We reconstructed mean fire interval of 5.5 years (MFI: all scars) and 3.6 years for the Weibull mean probability interval (WMPI). The MFI ( $\geq 25 \%$ scarred) was 8.9 years and WMPI was 6.9 years. The seasonal patterns of fire occurrence showed that most fires $(68.3 \%)$ were formed in middle earlywood, $30 \%$ in early earlywood and $1.7 \%$ in late earlywood. Considering the phenology of the species, it was determined that $98.3 \%$ of fires occurred in spring and $1.7 \%$ at the beginning of summer. The fires were recorded in dry years, but the relationship was not statistically significant. A strong relationship between droughts and widespread fires was observed. Likewise, it was determined that climate variability was strongly related to ENSO; fires reconstructed from 1956 to 2010 correspond with both El Niño and La Niña events.
\end{abstract}

Key words: Sierra de Manantlán, fire reconstruction, Pinus douglasiana, climate, El Niño Southern Oscillation.

\section{RESUMEN}

La Reserva de la Biosfera Sierra de Manantlán (RBSM) en Jalisco, representa la reserva más importante del Occidente de México, donde los incendios son uno de los principales elementos de la dinámica del bosque. Con el objetivo de reconstruir la historia del fuego, se colectaron secciones parciales de Pinus douglasiana con cicatrices de incendios. El fechado exacto de 293 cicatrices registradas en 51 árboles mediante técnicas dendrocronológicas, permitió reconstruir la frecuencia para el período 1867 a 2010 . El intérvalo medio de frecuencia (MFI, por sus siglas en inglés) fue de 5,5 años y un intérvalo medio de probabilidad de incendios (WMPI, por sus siglas en inglés) de 3,6 años, para todas las cicatrices. Al considerar cicatrices que se encuentran en $\geq 25 \%$ de las muestras, se determinó un MFI de 8,9 años y un WMPI de 6,9 años. La estacionalidad de ocurrencia indica, mayor cantidad de incendios (68,3\%) en la mitad de la madera temprana, $30 \%$ al inicio y $1,7 \%$ al final de la madera temprana. Considerando la fenología de la especie se determinó que el 98,3 \% de los incendios corresponde a la estación de primavera y el 1,7 \% al inicio del verano. Se observó relación entre la disminución de la lluvia (sequías) y la frecuencia de incendios severos, aunque no de manera significativa. Así mismo, se determinó que la variabilidad estuvo relacionada con El Niño Oscilación del Sur (ENSO), los incendios reconstruidos de 1956 a 2010 coinciden con eventos Niño y Niña.

Palabras clave: Sierra de Manantlán, reconstrucción de incendios, Pinus douglasiana, clima, El Niño Oscilación del Sur.

\section{INTRODUCCIÓN}

El fuego es un componente clave de la dinámica histórica de muchos ecosistemas terrestres y, particularmente, de los bosques del género Pinus (pinos) (Agee 1998). Aunque las especies de Pinus varían en sus adaptaciones a diferentes regímenes de incendios y condiciones ambientales variables (Keeley 2012), en las zonas de clima templado húmedo de las montañas del suroeste de los Estados Unidos y del norte de México predomina un régimen de incendios frecuentes, superficiales y de baja severidad, cuyo papel en la regulación de la estructura, composición de especies e incorporación de la biomasa muerta en los bosques de pino, ha sido ampliamente discutido y bien establecido en diferentes trabajos (Rodríguez-Trejo y Fulé 2003, Heinlein et al. 2005).

Los estudios de reconstrucción de la historia de incendios han sido empleados como una herramienta para analizar y entender la frecuencia y efectos de los incendios 
en diversos ecosistemas por muchos años (Grissino-Mayer 2010). Para el oeste de los Estados Unidos se ha logrado reconstruir la historia del fuego para los últimos 2000 años (Swetnam 1993) y 500 años para el norte de México (Stephens et al. 2003).

La información generada sobre los regímenes de incendios permite evaluar el comportamiento de este disturbio para regiones o sitios específicos y seleccionar las estrategias apropiadas de manejo del fuego (Fulé y Covington 1999). Estudios de regímenes de incendios en México, han sido desarrollados en bosque de pino, pino-encino y en bosques mixtos de coníferas en la zona fronteriza entre México y los Estados Unidos (Fulé et al. 2012, Poulos et al. 2013), en la Sierra Madre Oriental (Yocom et at. 2010) y en la Sierra Madre Occidental (Heyerdahl y Alvarado 2003, Fulé et al. 2005, Cerano-Paredes et al. 2010, Fulé et al. 2011). Aunque existen algunos estudios preliminares sobre la frecuencia histórica de incendios en bosques de pino de México al sur del Trópico de Cáncer (Rubio 2006, Cassell 2012, Llamas 2013), actualmente solo se ha publicado una cronología de incendios para los pinares de alta montaña del Pico de Orizaba, Veracruz (Yocom y Fulé 2012).

Con el objetivo de extender este tipo de trabajos en el centro-occidente del país, se seleccionó la Reserva de la Biosfera Sierra de Manantlán (RBSM), localizada en los límites de los estados de Jalisco y Colima. Esta reserva es una de las áreas protegidas más importante del Occidente de México, debido a su gran extensión (139.500 ha), alta riqueza de especies (más de 2.900 especies de plantas vasculares y 580 especies de vertebrados) (INE 2000), potencial forestal y servicios ambientales que presta a una extensa región de Colima y sur de Jalisco (Jardel 1992). Sus cuencas abastecen de agua los valles agrícolas y centros urbanos adyacentes, que albergan una población de más de 400.000 habitantes (INE 2000).

La Sierra de Manantlán tiene una larga historia de influencia humana en la configuración y dinámica del paisaje, a través de la agricultura y el uso del fuego (Jardel 1992); este último factor ha estado presente por lo menos en los pasados 4.200 años (Figueroa-Rangel et al. 2008). La ganadería extensiva desde el siglo XVII y la explotación comercial de madera durante la segunda mitad del siglo XX han sido factores importantes en la dinámica de las áreas boscosas (Jardel 1992).

Desde el establecimiento de la reserva de la biosfera en 1987, sólo en algunas áreas se ha seguido una política de supresión de incendios para favorecer la regeneración del bosque mesófilo de montaña (Jardel 2008), pero en general en los bosques de pino y encino del área se mantiene una incidencia de incendios relativamente alta (Balcázar 2011) y se ha puesto en marcha un plan de manejo del fuego (Jardel et al. 2006).

Dada la relevancia del área, la importancia del papel de los incendios en la dinámica del paisaje y la necesidad de fortalecer los fundamentos de las acciones de manejo del fuego en la RBSM, se plantearon varias preguntas de investigación: 1) ¿Cuál ha sido el régimen histórico de incendios en bosques de pino y cómo ha variado a través del tiempo? 2) ¿Se ha modificado el régimen histórico posterior al decreto de la reserva? y 3) ¿Existe una relación entre la variación del clima y los incendios en la RBSM? Se plantean dos hipótesis: 1) los regímenes del fuego se han modificado posterior al decreto de la reserva y 2) los incendios más severos se relacionan con eventos climáticos extremos (sequías) como parte de la variabilidad climática.

\section{MÉTODOS}

Área de estudio. La RBSM es un macizo montañoso con gran complejidad fisiográfica que se localiza en la Sierra Madre del Sur en sus intersecciones con la Sierra Madre Occidental y la Faja Volcánica Transmexicana (INE 2000). Presenta una cubierta vegetal variada que incluye desde selva baja caducifolia en las partes bajas con clima cálido subhúmedo hasta bosques de encino, pino y mesófilo de montaña en las partes altas con clima templado húmedo (INE 2000, Vélica-Zúñiga 2013).

El área de estudio Alto Las Joyas (ALJ) se ubica al centro oeste de la reserva, dentro de la Zona Núcleo Manantlán-Las Joyas (INE 2000), entre los $19^{\circ} 36^{\prime} 52,3^{\prime \prime} \mathrm{N}$ y $104^{\circ}$ $15^{\prime} 02,3^{\prime \prime} \mathrm{O}$ (figura 1). El gradiente de elevación oscila entre los 1.980 y 2.100 m s.n.m. El clima es templado cálido (temperatura media anual entre $15^{\circ}$ y $18{ }^{\circ} \mathrm{C}$ ), con una precipitación anual entre 1.200 y 1.800 mm, húmedo (razón de evapotranspiración potencial entre 0,5 y 1,0), con régimen de lluvias de verano e invierno-primavera secos (VélicaZúñiga 2013). La especie de conífera dominante en el sitio es Pinus douglasiana Martínez, mezclada con Pinus oocarpa Sciede ex Schlecht, Arbutus xalapensis Kunth, Quercus magnoliifolia Née y Quercus obtusata Humb. et Bonpl.

Métodos de campo. En una superficie de 40 ha ubicada en la parte alta de la RBSM, se seleccionaron de forma dirigida árboles con cicatrices de incendios. Se removieron secciones parciales de árboles vivos, muertos en pie, tirados y tocones empleando una motosierra Stihl modelo MS 660 (figura 2A). Se consideraron los individuos con el mayor número de registros de incendios (cicatrices), bien preservados y lo más longevos posible (Fulé et al. 2012).

Métodos de laboratorio. En el laboratorio, las muestras fueron montadas y pulidas con lijas de grano 40 a 1.200 para lograr una superficie uniforme y apreciar de manera clara las estructuras de crecimiento bajo microscopio (figura 2B y C). Las muestras fueron fechadas y se determinó el año exacto de formación de cada uno de los crecimientos anuales mediante la comparación de los patrones de crecimiento (Stokes y Smiley 1968). Una cronología maestra desarrollada con base a los crecimientos anuales de Pinus douglasiana para la RBSM fue también empleada como referencia para el correcto fechado de las muestras con cicatrices de incendios (Cerano-Paredes et al. 2013). 


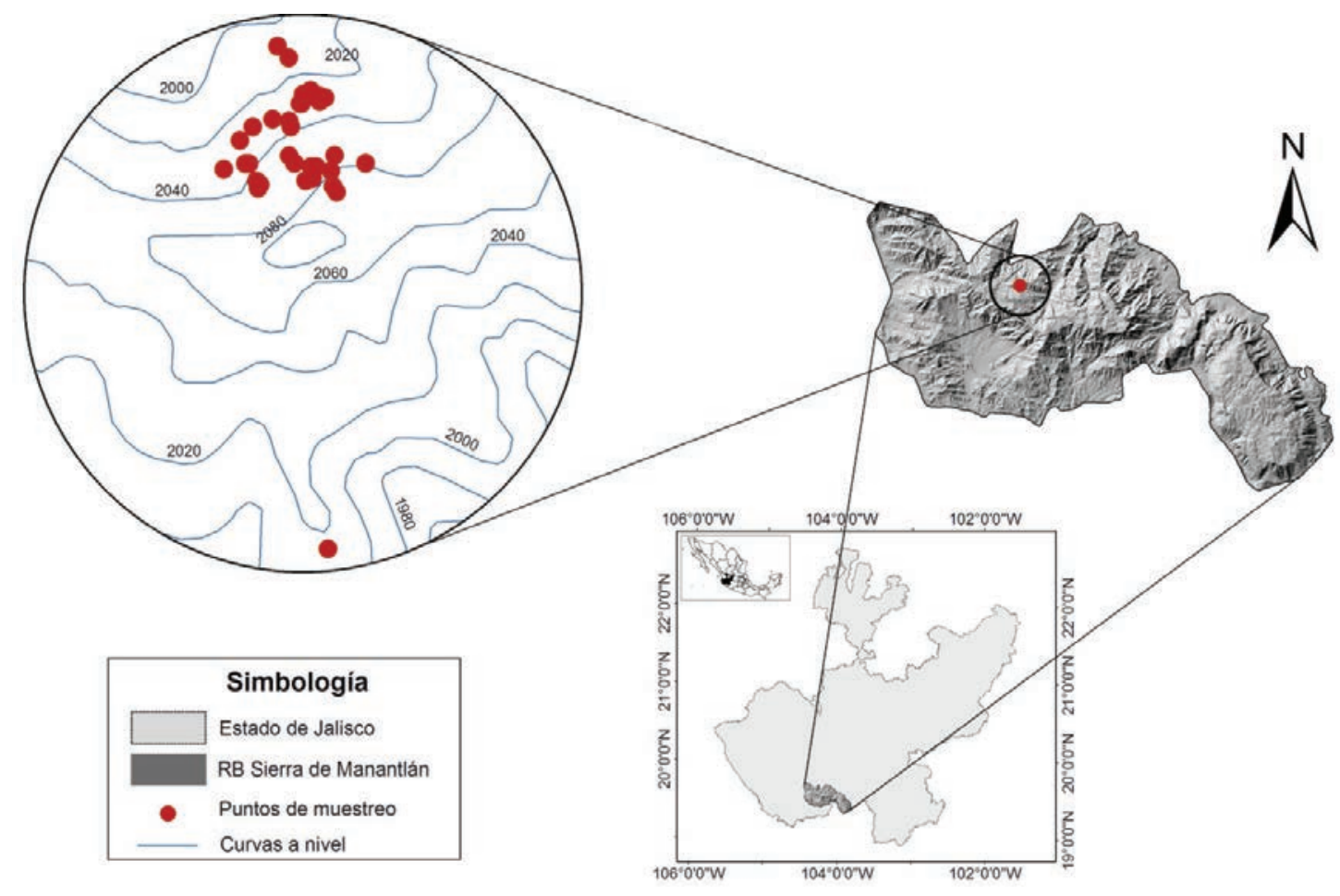

Figura 1. Localización geográfica del área de estudio (ALJ) dentro de la Reserva de la Biosfera Sierra de Manantlán (RBSM), Jalisco, al occidente de México.

Geographical location of the study area (ALJ) within Sierra de Manantlán Biosphere Reserve (RBSM), Jalisco, in western Mexico.

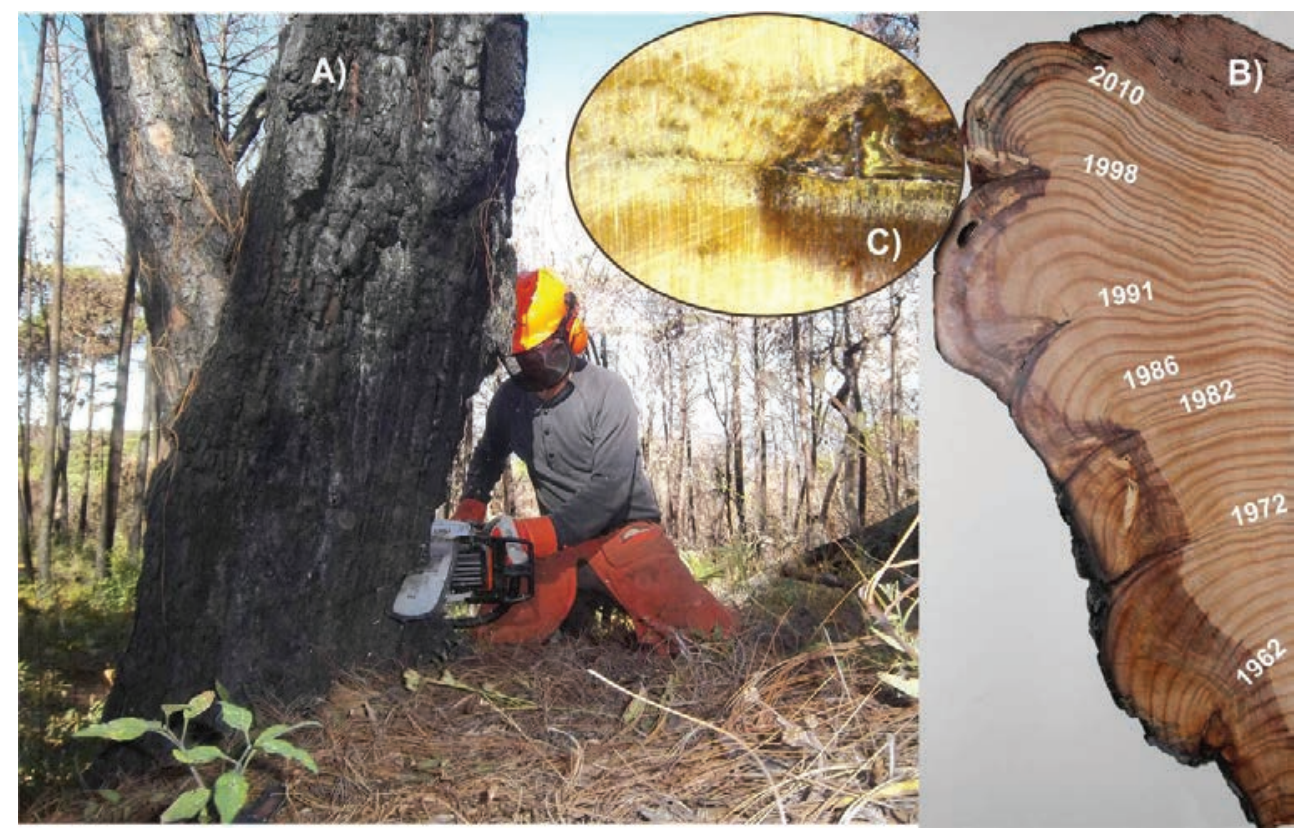

Figura 2. (A) Obtención de muestras con apoyo de motosierra en la Reserva de la Biosfera Sierra de Manantlán, Jalisco, (B) sección parcial de Pinus douglasiana con excelentes registros históricos de incendios (7 cicatrices) posterior a su preparación y fechado, y (C) muestra un acercamiento microscópico de la cicatrización del incendio de 1998, que permite definir la posición de la cicatriz en el anillo de crecimiento (mitad de la madera temprana) que define la época en el año en que se registró.

(A) Sampling supported by chainsaw in Sierra de Mananatlán Biosphere Reserve, Jalisco, (B) partial section of Pinus douglasiana with excellent records of fire history (7 scars) after preparation and dated, and (C) microscopic approach of healing fire 1998 that defines the position of the scar in the growth ring (middle earlywood) defining the time the year it was recorded. 
Después del fechado, el ancho de anillo de todas las muestras se midió con un sistema de medición Velmex de precisión $0,001 \mathrm{~mm}$. La calidad del fechado se verificó con el programa Cofecha (Holmes 1983). Las cicatrices de los incendios se emplearon como evidencia de incendios, identificados como una discontinuidad en las células en el anillo o lo largo del límite del anillo de crecimiento donde el cambium murió, seguido por una supresión y deformación de los crecimientos (Dieterich y Swetnam 1984).

La estación de ocurrencia de los incendios se estimó con base a la posición relativa de cada una de las cicatrices de los incendios en el anillo anual con base en las siguientes categorías: EE (inicio de la madera temprana), ME (mitad de la madera temprana), LM (final de la madera temprana), L (madera tardía) y D (dormancia o límite del anillo) (Dieterich y Swetnam 1984, Baisan y Swetnam 1990). Las diferentes categorías se agruparon en dos períodos: 1) primavera $(\mathrm{D}+\mathrm{EE})$ y 2$)$ verano $(\mathrm{ME}+\mathrm{LE}+\mathrm{L})$ (Grissino-Mayer 2001).

Análisis de datos. La base de datos de historia del fuego se analizó con el programa FHX2, versión 3.2 (Grissino-Mayer 2001). El análisis consideró dos períodos: 1) inicio en el año 1867, correspondiente al primer registro de incendio, y término en el año 2010; y 2) inicio a partir del primer año con un adecuado tamaño de muestra (1960), definido por el primer año de incendio registrado en el $10 \%$ o más del número total de muestras (Grissino-Mayer et al. 1994) y finalizó en el año 2010.

Los intervalos medios de frecuencia y recurrencia de incendios se analizaron estadísticamente en función de tres filtros diferentes: (1) todas las cicatrices, (2) cicatrices registradas en $\geq 10 \%$ de las muestras y (3) cicatrices registradas en $\geq 25 \%$ de las muestras. El filtro de $25 \%$ o más, es utilizado comúnmente como un estimador de la frecuencia de incendios, que incluye aquellos incendios que fueron relativamente extensos e intensos (Fulé y Covington 1999, Swetnam y Baisan 2003).

Para cada uno de los filtros, se determinaron las siguientes estadísticas descriptivas: intervalo medio de frecuencia (MFI, por sus siglas en inglés), intervalo mínimo y máximo, y el intervalo de probabilidad media de Weibull (WMPI, por sus siglas en inglés). Esta última es una medición de distribución central, empleada para modelar la distribución asimétrica de los intervalos de incendios y para expresar en términos probabilísticos los intervalos de recurrencia (Grissino-Mayer et al. 1994).

Relación clima-incendios. Para analizar las condiciones climáticas y su relación en la frecuencia de incendios se empleó el análisis de sobreposición de época (SEA, por sus siglas en inglés) del programa FHX2 versión 3.2 (GrissinoMayer 2001). Como proxy del clima se empleó una cronología de ancho de anillos de Pinus douglasiana. La versión residual de esta cronología explicó 52 \% de la variabilidad de la precipitación regional invierno-primavera y cubre el período 1792-2010, donde se registraron sequías severas, especialmente en 1850, 1860 y 1950 (Cerano-Paredes et al. 2013). También se utilizaron los índices Niño 3.4 de El Niño Oscilación de Sur (ENSO, por sus siglas en inglés) (Trenberth 1997). Ambas variables se compararon durante el incendio, cinco años anteriores y dos años posteriores al mismo. Para evaluar la significancia estadística del análisis SEA, los intervalos de confianza $(95,99$ y $99,9 \%)$ se calcularon utilizando la distribución bootstrapped de los datos climáticos con 1.000 repeticiones. Asi mismo, se compararon de manera gráfica las series de historia de incendios y la reconstrucción de precipitación invierno-primavera (Cerano-Paredes et al. 2013), para determinar la relación entre eventos extremos en disminución de lluvia y la frecuencia de incendios en años específicos a través del tiempo.

\section{RESULTADOS}

Historia de incendios. Se colectó un total de 55 secciones parciales con cicatrices de incendios; 37 (67\%) de árboles muertos y 18 (33 \%) de vivos. Se logró fechar un total de 51 muestras (93\%), las cuales fueron empleadas en este análisis. Todas las secciones colectadas fueron de Pinus douglasiana, donde el diámetro promedio de los árboles fue de $67,6 \mathrm{~cm}$.

Se fechó un total de 293 cicatrices, la estación de ocurrencia fue determinada para el $100 \%$ (cuadro 1). No se registró ninguna cicatriz ( $0 \%$ en la estación de dormancia, al inicio de la madera temprana se determinó $30 \%$ de los incendios, la mayor cantidad de cicatrices $(68,3 \%)$ se registró en la mitad de la madera temprana (figura 2C), sólo el 1,7 \% de las cicatrices se observó al final de la madera temprana y ninguna cicatriz en la madera tardía. El resultado del análisis de estacionalidad, considerando la fenología de Pinus douglasiana para esta región de México (cuadro 1), indicó que el mayor porcentaje de incendios $(98,3 \%)$ se presentó en primavera y una menor proporción $(1,7 \%)$ en verano.

Aunque el sitio tuvo muestras cuyos primeros anillos se fecharon antes de 1850 (específicamente 1838), el primer incendio se registró en el año 1867 (figura 3). En el primer período completo de análisis correspondiente al intervalo 1867-2010, los incendios han sido frecuentes en esta parte de la RBSM (figura 3). El MFI calculado en la categoría que considera todas las cicatrices fue de 5,5 años, mientras que el WMPI para la misma categoría fue de 3,6 años (cuadro 2). Al considerar las cicatrices observadas en el $10 \%$ o más de las muestras, se determinó un MFI de 6,2 años y un WMPI de 4,2. Para las cicatrices registradas en la categoría de $25 \%$ o más de las muestras, el MFI incremento a 8,9 años y el WMPI fue de 6,9 años. Los incendios han registrado una frecuencia media de cinco años y dentro de estos eventos, los episodios más severos tuvieron una recurrencia de nueve años.

Al considerar los últimos 50 años (período 1960-2010), los incendios más severos (registrados simultáneamente en al menos $25 \%$ de las muestras) indican estadísticos de 
Cuadro 1. Distribución de la estacionalidad de las cicatrices de incendios (número y porcentaje) basado en la posición de la cicatriz del incendio en el anillo anual de crecimiento (D, dormancia; EE, inicio de madera temprana; ME, mitad de la madera temprana; LE, final de la madera temprana y L, madera tardía).

Distribution of the seasonality of fire scars (number and percentage) based on the position of the fire scar in the annual growth ring (D, dormancy, EE, early earlywood, ME, middle earlywood, LE, late earlywood and L, latewood).

\begin{tabular}{lcccccccc}
\hline \multicolumn{1}{c}{ Cicatrices } & $\begin{array}{c}\text { Estación } \\
\text { determinada }\end{array}$ & D & EE & ME & LE & L & $\begin{array}{c}\text { Incendios de } \\
\text { primavera }^{\mathrm{A}}\end{array}$ & $\begin{array}{c}\text { Incendios de } \\
\text { verano }^{\mathrm{B}}\end{array}$ \\
\hline Número & 293 & 0 & 88 & 200 & 5 & 0 & 288 & 5 \\
Porcentaje & 100 & 0,0 & 30,0 & 68,3 & 1,7 & 0,0 & 98,3 & 1,7 \\
\hline
\end{tabular}

Nota: La definición de la estacionalidad de los incendios para esta región del país fue con base en la fenología de la especie.

${ }^{\mathrm{A}}$ Dormancia + inicio de madera temprana + mitad de la madera temprana (D+EE+ME).

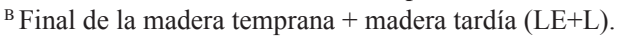

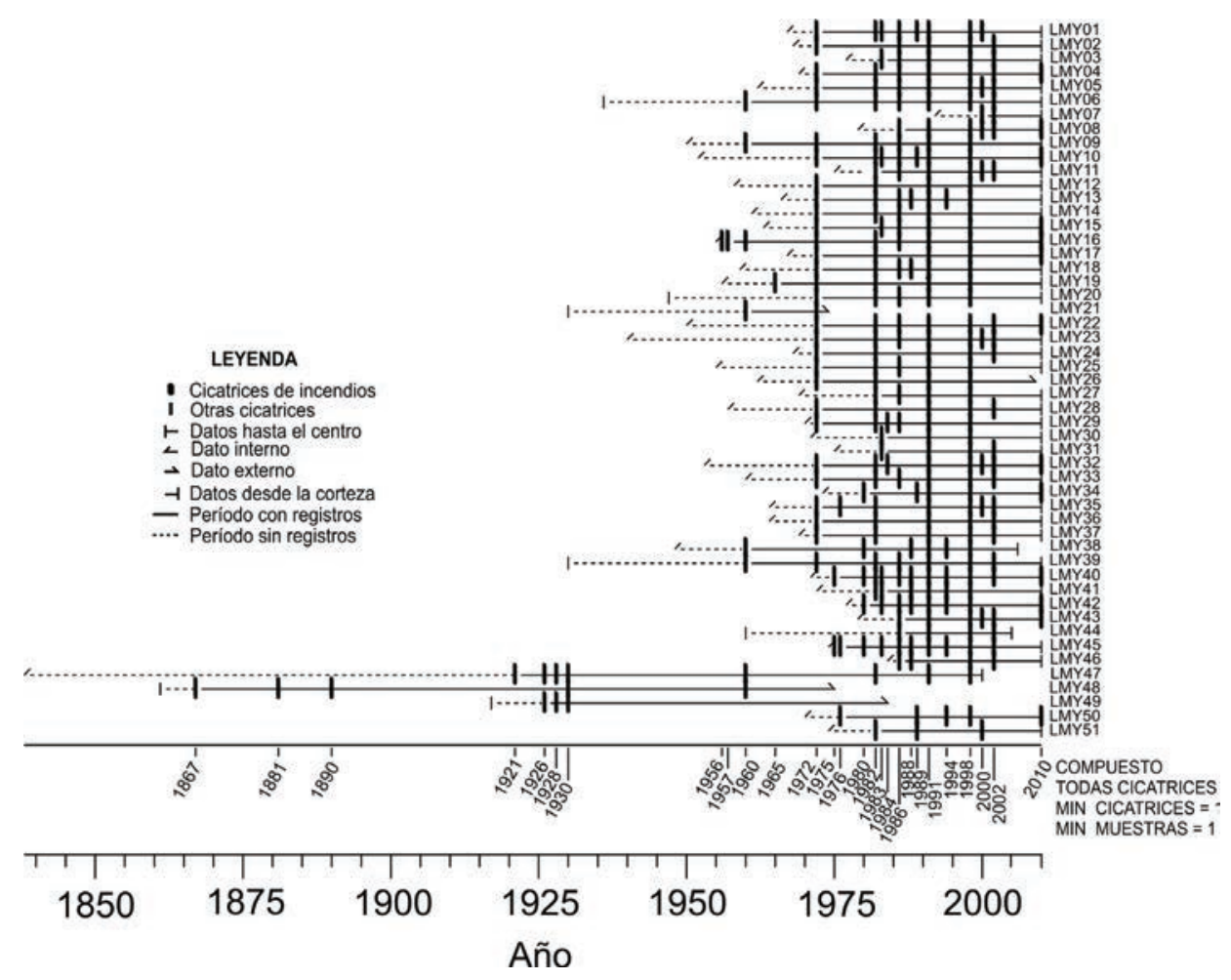

Figura 3. Reconstrucción de la historia de incendios (1838-2010) en la RBSM, Jalisco, considerando todas las cicatrices. Las líneas horizontales representan la longitud de cada uno de los árboles con cicatrices de incendios, punteadas antes del primer registro de incendio y sólidas en todo el período con registros. Las barras verticales resaltadas en color negro representan las cicatrices de los incendios. Los años en los cuales se registraron cada uno de los incendios se muestran en el eje secundario de las X.

Fire history reconstruction (1838-2010) in RBSM, Jalisco, considering all scars. The horizontal lines represent the length of each of the trees with fire scars, dotted before the first fire record and solid throughout the period with records, vertical bars highlighted in black represent the firescars. The year in which each fire is recorded is on the secondary axis of the $\mathrm{X}$.

MFI y WMPI similares a los obtenidos para el período total (7,14 y 7,05, respectivamente) (cuadro 2). Sin embargo, al considerar las frecuencias para todas las cicatrices y las registradas en al menos $10 \%$ de las muestras, los estadísticos (MFI y WMPI) presentaron intervalos de tiempo más cortos (cuadro 2).
De las 293 cicatrices de incendios datadas, se determinó un total de 27 incendios en el período de 173 años, entre 1838-2010, en función del registro más extenso (figura 3). $\mathrm{Al}$ aplicar el filtro $\geq 25 \%$ que permite distinguir aquellos eventos más fuertes, se observó que 17 (63\%) de los 27 incendios fueron severos (figura 4). 
Cuadro 2. Estadísticas de los intervalos de distribución de los incendios para el período 1867-2010 y 1960-2010 en la RBSM, Jalisco, México.

Statistic of fires distributions intervals for the period 1867-2010 in the RBSM, Jalisco, Mexico.

\begin{tabular}{|c|c|c|c|c|c|}
\hline Período de análisis & Categoría de análisis & MFI & Min. & Max. & WMPI \\
\hline \multirow{3}{*}{$1867-2010$} & Todas las cicatrices & 5,50 & 1 & 31 & 3,63 \\
\hline & $10 \%$ Cicatrices & 6,22 & 1 & 31 & 4,25 \\
\hline & $25 \%$ Cicatrices & 8,94 & 1 & 31 & 6,95 \\
\hline \multirow{3}{*}{$1960-2010$} & Todas las cicatrices & 2,94 & 1 & 8 & 2,63 \\
\hline & $10 \%$ Cicatrices & 3,57 & 1 & 8 & 3,19 \\
\hline & $25 \%$ Cicatrices & 7,14 & 4 & 12 & 7,05 \\
\hline
\end{tabular}

Notas: El análisis estadístico fue realizado en tres categorías: (a) considerando todas las cicatrices de incendios, incluyendo aquellas muestras con un solo registro, (b) fueron considerados los años de incendios registrados en el $10 \%$ o más de las muestras y (c) únicamente se consideró el registro de incendios observados en el $25 \%$ o más de las muestras analizadas. MFI, intervalo medio de frecuencia; Min, mínimo; Max, máximo; WMPI, intervalo medio de probabilidad de Weibull.

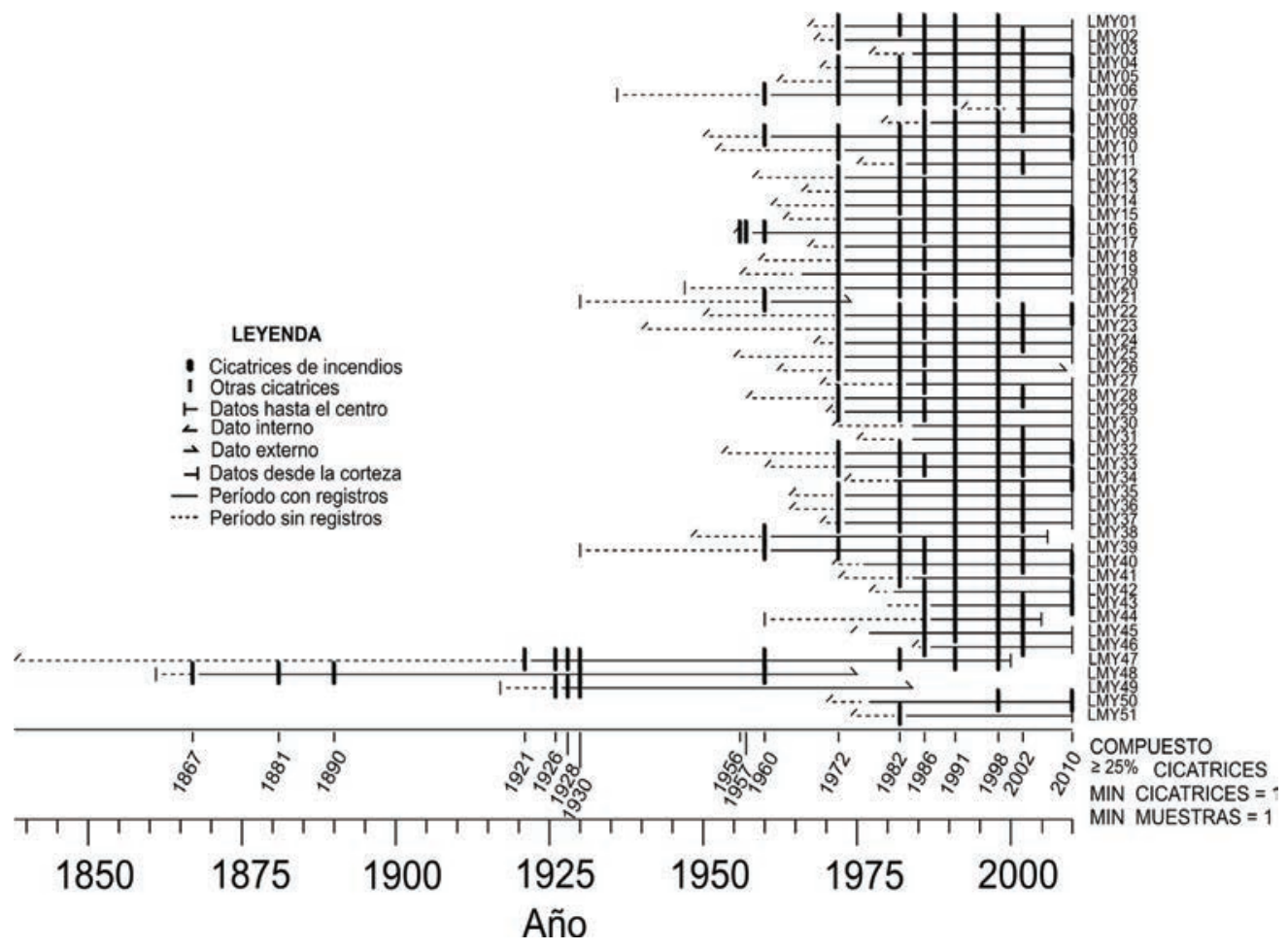

Figura 4. Reconstrucción de la historia de incendios en la RBSM, Jalisco, considerando el $\geq 25 \%$ o más de las cicatrices registradas en el total de las muestras, incendios de alta intensidad.

Fire history reconstruction in the RBSM, Jalisco, considering the $25 \%$ or more of the registered scars in all samples, fires of high intensity.

Relación clima-incendios. El análisis de sobreposición de época (SEA), tanto para todos los incendios como los registrados en el $25 \%$ del total de las muestras, indica valores de clima regional (índice de ancho de anillo) por debajo de la media durante el incendio y valores del Niño 3,4 apenas cercanos a la media (figura 5). Estas tendencias no fueron significativas $(P>0,05)$ en función de las simulaciones generadas por SEA. Sin embargo, para los incendios más ex- tensos $(\geq 25 \%$ de las cicatrices registradas en el total de las muestras) el año inmediatamente anterior presentó valores del índice Niño 3,4 significativamente $(P<0,01)$ por debajo del promedio, mientras que el año posterior mostró valores superiores al promedio $(P<0,05)$. Específicamente para los incendios de los años 1972, 1982, 1986, 1991 y 1998, que registran los más extensos y severos, los valores de precipitación estuvieron por debajo de la media (figura 6), influen- 

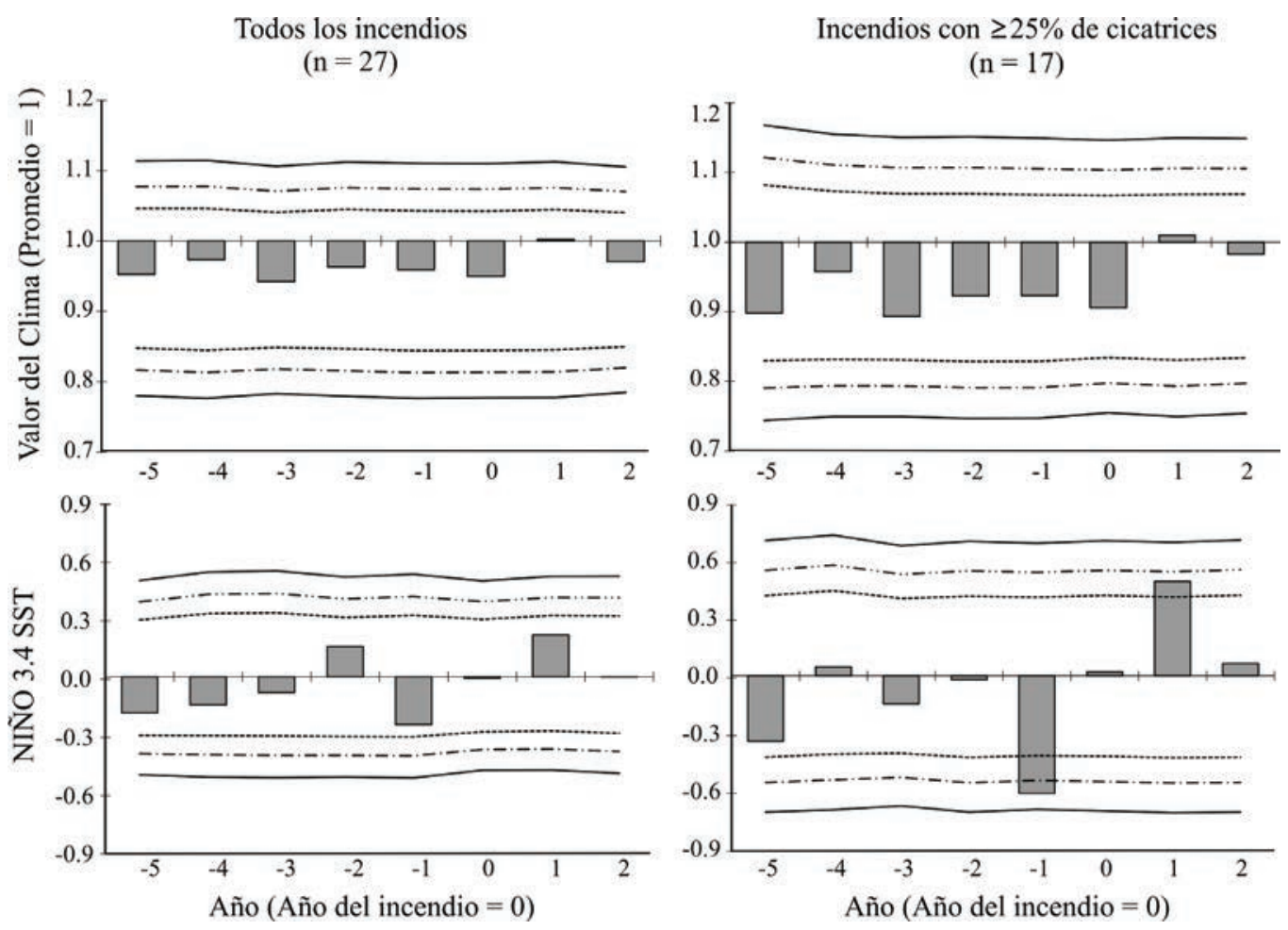

Figura 5. Análisis de Sobreposición de Época que muestra la relación entre la ocurrencia del incendio con el clima regional (índice de ancho de anillo) e índice de ENSO Niño 3,4, para todos los incendios e incendios registrados en el $\geq 25 \%$ de las muestras. El año del incendio está indicado por el 0, condiciones cinco años antes del incendio (valores negativos) y dos años después del incendio (valores positivos). Las tres líneas superiores e inferiores representan los intervalos de confianza al 95 \%, $99 \%$ y $99,9 \%$.

Superposed Epoch Analysis showing the relationship between fire occurrence with regional climate (tree-ring with index) and NIÑO 3.4 ENSO index, for all fires years and fire years in which $\geq 25 \%$ of samples were scarred. The fire year is indicated by 0 , conditions five years before the fire (negative values) and two years after the fire (positive values). The upper and lower three lines represent the confidence intervals at $95 \%, 99 \%$ and $99.9 \%$.

ciados por eventos de ENSO. En general, este fenómeno tanto en su fase El Niño como La Niña, contribuyó a modular la frecuencia de incendios.

\section{DISCUSIÓN}

Regímenes del fuego. En este estudio se logró reconstruir la historia de incendios en la RBSM para los últimos 143 años (1867-2010). La extensión de la reconstrucción generada, obedece a que no se encontraron árboles longevos en el área, atribuible al intenso aprovechamiento forestal ocurrido en el período de 1940 a 1980 (Jardel 1998). Los incendios reconstruidos entre 1867 y 1940 (figura 4) se obtuvieron de secciones transversales de tocones bien conservados y con cicatrices de incendios. La baja frecuencia de incendios detectados en el período 1931-1971 (figura 4) se atribuye, por un lado, al intenso aprovechamiento forestal que eliminó la mayor cantidad de arbolado grande con posibles registros de incendios y por otro debido a un mayor control del fuego durante la etapa de aprovechamiento maderero.

Se esperaba encontrar un cambio en la frecuencia de incendios posterior al decreto de la reserva de 1987. Sin embargo, la presencia de incendios superficiales posterior a 1987, ha sido constante y no se observa una interrupción en los últimos años. El MFI (1867-2010) fue de 5,5 años para todas las cicatrices y de 8,9 años para los incendios más severos, y el WMPI de 3,6 y 6,9 años para las categorías de incendios respectivas. Rubio (2006) dentro del vecino ejido Ahuacapán, determinó un intervalo medio entre incendios de 4,4 a 7,3 años. Estudios recientes cercanos a nuestra área de trabajo, desarrollados por Cassell (2012) y Llamas (2013) reportan un MFI de 5,5 y cinco años (todas las cicatrices) y un WMPI de cinco y 4,8 años, respectivamente.

Los bosques de la reserva han estado sujetos a una alta frecuencia de incendios superficiales, con un intervalo común de cinco años entre las diferentes áreas, lo que es atribuible a que condiciones climáticas similares afectan una amplia superficie de la reserva. Resultados similares se han reportado para bosques de pino del norte de México (Fulé y Covington 1999, Heyerdahl y Alvarado 2003, Fulé et al. 2005, 2011, Yocom et al. 2010).

La sincronía de los MFI en la RBSM obedece a que los estudios realizados han determinado los estadísticos considerando el período total de registro. Sin embargo, al 


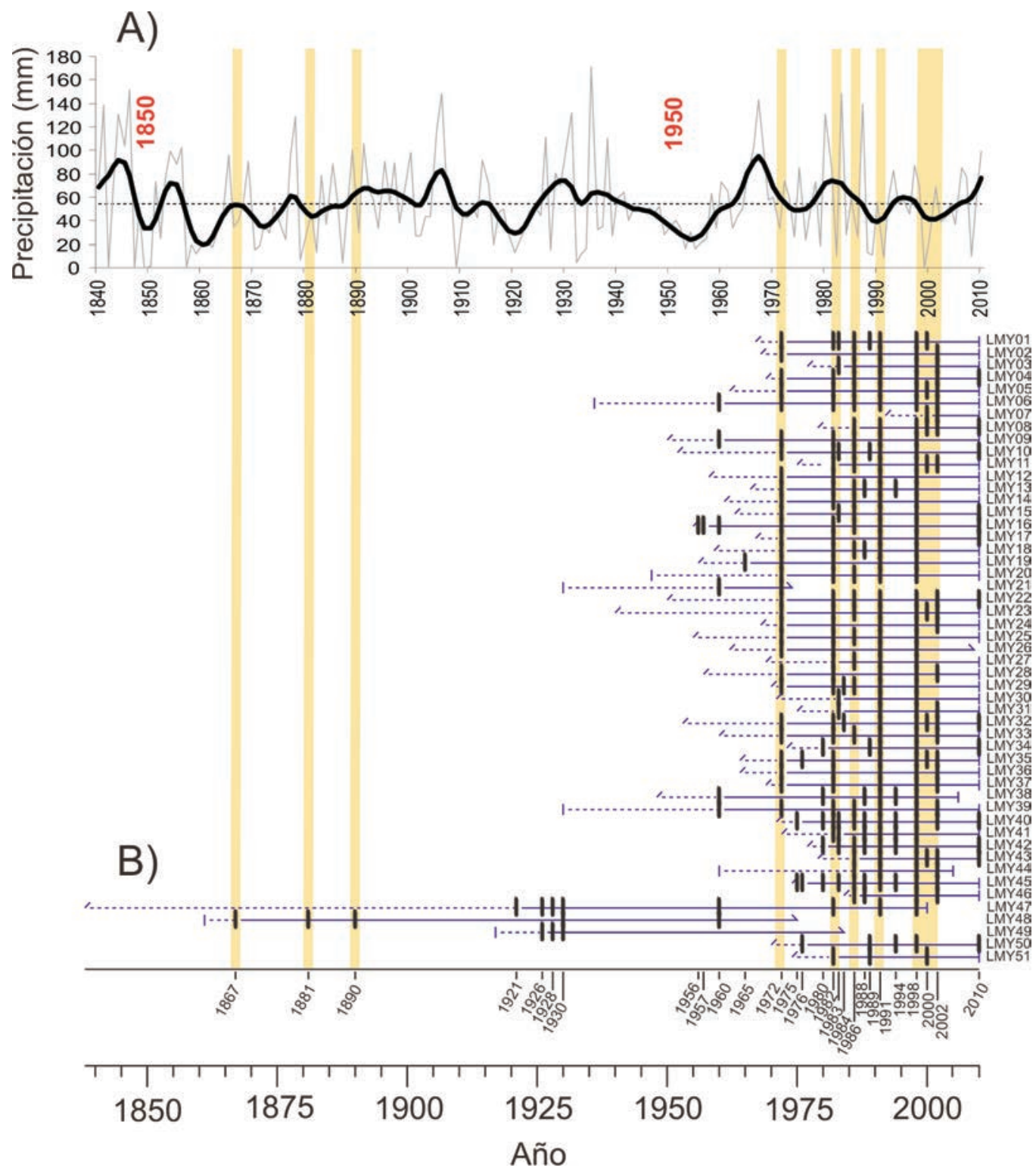

Figura 6. Relación entre la variabilidad climática y la frecuencia histórica de los incendios en la Reserva de la Biosfera Sierra de Manantlán, Jalisco. (A) Representa la precipitación reconstruida invierno-primavera para la RBSM (Cerano-Paredes et al. 2013), la línea gris de fondo representa la variabilidad anual, la resaltada en color negro es una curva flexible con escala de 10 años para analizar mejor eventos secos y húmedos, la línea horizontal punteada indica la precipitación promedio regional y (B) representa la reconstrucción del historial del fuego. Las líneas verticales en color amarillo permiten analizar la relación entre la frecuencia de los incendios y la disminución de la precipitación por debajo del promedio (sequías).

Relationship between the climate variability and the historical frequency of fires in Sierra de Manantlan Biosphere Reserve, Jalisco. (A) Represents winter-spring precipitation reconstructed for RBSM (Cerano-Paredes et al. 2013), the bottom gray line represents the annual variability, highlighted in black is a flexible curve with scale of 10 years to better analyze dry and wet events. The horizontal dashed line indicates regional average precipitation and (B) represents the fire history reconstruction. The yellow vertical line allows analyzing the relationship between fire frequency and decreasing precipitation below average (droughts).

considerar el tamaño de muestra estadísticamente adecuado (1960-2010) los intervalos de frecuencia varían, registrando 2,94 años y 3,57 años, para incendios de baja y mediana intensidad, respectivamente (cuadro 2). Para fines de manejo se deben considerar los intervalos de frecuencia determinados para ambos períodos de análisis.
Más del $60 \%$ de los incendios registrados en el área de estudio se clasifican como incendios superficiales (figura 4), por registrarse en un alto porcentaje de las muestras, condición que determina su extensión e intensidad (Fulé y Covington 1999, Swetnam y Baisan 2003). Para el período 1960-2010 donde los incendios se registran en un alto nú- 
mero de muestras esto se cumple. Por el contrario, para el período 1850-1950 esto no se observa, atribuido al reducido número de árboles longevos en el sitio, lo que determinó que un solo incendio represente el $100 \%$ de los eventos, quedando indicado como incendio severo. Sin embargo, los incendios de 1926, 1928 y 1930 indican sincronía en las muestras existentes, lo cual puede indicar que estos incendios pudieron haber tenido un efecto similar a los más recientes. Considerando la diferencia en el número de incendios para ambos intervalos de tiempo los estadísticos MFI y WMPI se generaron para ambos períodos (cuadro 2).

Las estadísticas sobre incendios en la RBSM para el período 1995 a 2008 (Balcázar 2011), reportan los años de 1998 y 2002 como los dos eventos más intensos y con mayor superficie afectada en la zona (15.237 y 6.572 ha, respectivamente). Esta información corrobora el historial reconstruido, donde se observa la severidad de ambos eventos, en particular, el incendio de 1998 registrado en el $96 \%$ de las muestras que se extienden hasta esta fecha (figura 4).

Estacionalidad de los incendios. La distribución estacional de los incendios, basada en la posición relativa de cada una de las cicatrices en el anillo anual se determinó con base en la metodología propuesta por Dieterich y Swetnam (1984) y Baisan y Swetnam (1990), la mayoría de los incendios se categorizaron en la mitad de la estación de crecimiento (ME: 68,3\%) y un menor porcentaje al inicio (EE: $30 \%$ ) y final de la estación de crecimiento (LE: 1,7 \%). Los incendios de primavera ocurren entre finales de abril e inicio de junio, lo cual corresponde a un período seco, que se extiende en primavera; mientras que los incendios registrados en verano, ocurren entre finales de junio e inicio de septiembre (Swetnam et al. 2001).

Se determinó un $30 \%$ de los incendios en primavera y un $70 \%$ en la época de verano. Sin embargo, Jardel (2006) reporta que los incendios forestales en la RBSM se presentan en la temporada seca del año, entre finales de diciembre y principios del mes de junio. Este hecho corrobora el porcentaje de incendios $(30 \%)$ determinado en primavera. Por el contrario, el $70 \%$ de los incendios identificados para verano, no presentan una sincronía con la estación seca. Swetnam et al. (2001) señala que la estacionalidad de los incendios está determinada por la zona geográfica y la fenología de la especie principalmente. El crecimiento de Pinus douglasiana en la RBSM está influenciado por la lluvia estacional de invierno-primavera (Cerano-Paredes et al. 2013), el crecimiento del anillo está desarrollado casi en su totalidad para el mes de julio. De esta manera, se puede inferir que los incendios categorizados en ME (cuadro 1) ocurrieron entre finales de abril e inicios de junio, correspondiendo a la parte final de la estación de primavera, período seco y en el que se registra el mayor porcentaje de incendios en la zona. El $30 \%$ de los incendios categorizados en primavera posiblemente tuvieron lugar en los primeros meses del año y el 68,3 \% correspondientes a ME ocurrieron al final de la primavera.
En general se puede concluir que, en su mayoría, los incendios corresponden a la estación de primavera (98,3 \%) (cuadro 1), similar a lo reportado por Cassell (2012) y Llamas (2013). Sin embargo, se difiere de estas autoras, en lo referente a la categorización de las cicatrices dentro del anillo anual que definen la estacionalidad de los incendios.

Continuidad de la frecuencia de incendios. La mayoría de los árboles en RBSM muestran una frecuente ocurrencia de incendios hasta el 2010, con lo cual se da respuesta a nuestra segunda pregunta de investigación y se rechaza la hipótesis 1 que proponía que posterior al decreto de la reserva se había modificado la frecuencia de incendios. El intervalo libre de incendios más reciente fue de ocho años correspondiente al período 2002 a 2010. Se han generado diversos trabajos de la historia del fuego que reportan resultados similares, una ininterrumpida frecuencia de los incendios para el norte de México (Heyerdahl y Alvarado 2003, Cerano-Paredes et al. 2010, Fulé et al. 2011) y para el centro del país (Yocom et al. 2012).

En las áreas forestales de México, la interrupción de la frecuencia de incendios es muy variable, las historias de incendios para el norte del país presentan diferentes períodos de exclusión, 1930 y 1940 (Fulé y Covington 1999, Yocom et al. 2010), 1950 (Heyerdahl y Alvarado 2003, Fulé et al. 2005) y 1970 (Fulé y Covington 1999, CeranoParedes et al. 2010). Este patrón del norte de México se diferencia del suroeste de los Estados Unidos, donde se ha reportado una exclusión de incendios desde principios del siglo XIX (Swetnam y Baisan 2003) y finales del mismo siglo (Grissino-Mayer y Swetnam 2000, Heinlein et al. 2005), bosques con 100 y 200 años libres de incendios.

Relación clima-incendios. La falta de una asociación significativa $(P>0,05)$ entre la frecuencia de incendios y la cronología de ancho de anillo, proxy climático de la región y ENSO (figura 5), se atribuye a la corta escala de tiempo y el reducido número de fechas de incendios contempladas en el análisis SEA. Sin embargo, se determinó que los incendios más extensos se registraron en condiciones de años con sequías extremas, después de varios años consecutivos con condiciones ambientales limitantes y valores del índice Niño 3,4 que apenas alcanzaron la media (figura 5) precedidos de un año con valores significativamente por debajo de la media $(P<0,01)$ (figura 5$)$. La presencia de incendios se vio favorecida por condiciones La Niña años antes del incendio $(P<0,01)$ y eventos El Niño con una precipitación invernal no significativa $(P>0,05)$.

Al relacionar la serie de variabilidad de precipitación (invierno-primavera) generada para esta zona (CeranoParedes et al. 2013) y la reconstrucción de incendios para el período común de 1840-2010 (figura 6), se observa una relación positiva entre la disminución de la lluvia, relacionada con sequías y la frecuencia de incendios más severos. Asi mismo, esta variabilidad está relacionada con eventos extremos ENSO (figura 6). Cada uno de los incendios 
reconstruidos de 1956 a 2010 coinciden con eventos del ENSO tanto en su fase El Niño como La Niña. Englehart y Douglas (2001) han reportado la importancia del ENSO en las condiciones climáticas del oeste de México.

Trenberth (1997) reporta eventos ENSO en su fase El Niño y La Niña para el período de 1950-1996. Los incendios reconstruidos para los años 1956, 1972, 1975, 1976, 1984, 1988 y 1989 (figura 3) coincidieron con eventos La Niña que propició la disminución de las lluvias en la región (figura 6). Por el contrario, los incendios de 1957, 1980, 1982, 1983, 1986, 1991 y 1994 coincidieron con eventos El Niño (figura 3) que no produjeron un incremento significativo en la precipitación invernal (figura 6).

Los eventos Niño, en algunos casos, ocurrieron posterior de eventos intensos de La Niña, registrando una precipitación no significativa, lo que propicia condiciones favorables para la presencia de incendios. Por ejemplo, (1) La Niña de junio de 1954 a marzo de 1956, 22 meses de sequía (Trenberth 1997), originó la presencia de incendios en 1956 y El Niño de 1957 no registró lluvias invernales que rebasarán la media favoreciendo la ocurrencia de incendios (figura 6). (2) La Niña que inició en mayo de 1988 a junio de 1989, 14 meses de condiciones secas (Trenberth 1997), que aunado a las bajas condiciones de lluvia en 1990 (figura 6) y lluvias invernales de 1991, atribuibles estas últimas a El Niño, crearon las condiciones ideales para la propagación de incendios severos en 1991.

El incendio de 1998 representa uno de los más extensos reconstruidos para la RBSM; estadísticas del área lo categorizan como el año con mayor superficie afectada por incendios (15.237 ha) (Balcázar 2011). El Niño de 1998 es considerado uno de los eventos más severos que impactó el centro y sur de México, considerado un año record en la presencia de incendios (Rodríguez-Trejo y Pyne 1999). Asi mismo, el incendio más reciente reconstruido para el año 2010 está relacionado con un evento intenso El Niño (NOAA 2014). En la RBSM se ha reportado una influencia significativa $(P<0,05)$ del ENSO en la variabilidad de la lluvia invernal (Cerano-Paredes et al. 2013) y en la incidencia de incendios de alta intensidad (Jardel et al. 2006, Balcázar 2011).

Los resultados reportados en este estudio dan respuesta a la tercera pregunta de investigación y validan nuestra segunda hipótesis de que los incendios de mayor intensidad se ven en alto grado modulados por eventos climáticos extremos.

\section{CONCLUSIONES}

En la RBSM, la reconstrucción del régimen histórico de incendios para los últimos 144 años (1867-2010) muestra que el fuego registra una frecuencia media de 5,5 años y una recurrencia de nueve años para los eventos más severos. Se observa una ininterrumpida ocurrencia de estos eventos posterior al decreto de la reserva. Así mismo, se aprecia una relación significativa entre la disminución de la lluvia (sequías) y la incidencia de incendios.

Se rechaza la primera hipótesis que establece un cam- bio en la frecuencia de incendios posterior al decreto de la reserva, ya que se observa una incidencia ininterrumpida de incendios. Pero se acepta la segunda hipótesis, que los incendios más severos están relacionados con fuertes sequías, donde eventos ENSO han propiciado una disminución significativa de la precipitación un año anterior a los incendios y condiciones de lluvia no significativa durante el incendio, lo que ha modulado su frecuencia histórica.

Los resultados de esta investigación son de gran relevancia para los administradores de la reserva, que pueden hacer uso de este conocimiento para mantener o restaurar los regímenes históricos de incendios en el futuro con miras a garantizar la conservación del vasto recurso biológico del área. La Reserva de la Biosfera Sierra de Manantlán representa una zona muy extensa con diferentes condiciones climáticas y de vegetación, por tal razón es importante continuar desarrollando este tipo de estudios en los diferentes ecosistemas, que permitan obtener información específica para lograr una mejor planeación del manejo y control del fuego.

\section{AGRADECIMIENTOS}

Esta investigación fue posible gracias al financiamiento SEP-CONACYT a través del proyecto "Estudio de la relación clima-incendios en el norte-centro de México". Gracias a la Dirección de la RBSM-CONANP y a la Estación Científica Las Joyas de la Universidad de Guadalajara por permitirnos el acceso. Ernesto Rubio Camacho, Vicenta Constante García, Omar Duran Guerra y Luis Ubaldo Castruita, gracias por su apoyo en el trabajo de campo.

\section{REFERENCIAS}

Agee JK. 1998. Fire and pine ecosystems. In Richardson D ed. Ecology and biogeography of Pinus. Cambridge, UK. Cambridge University Press. p. 193-218.

Baisan CH, TW Swetnam. 1990. Fire history on a desert mountain range: Rincon Mountain Wilderness, Arizona, U.S.A. Canadian Journal of Forest Research 20: 1559-1569.

Balcázar MOE. 2011. Patrones geoecológicos de incendios forestales en la Reserva de la Biosfera Sierra de Manantlán. Tesis de Maestría en Ciencias en Manejo de Recursos Naturales. Autlán, Jalisco, México. Departamento de Ecología y Recursos Naturales, Universidad de Guadalajara. 145 p.

Cassell BA. 2012. Fire history of the Sierra de Manantlán Reserve in western México. Thesis Master of Science. Washington, USA. University of Washington. $96 \mathrm{p}$.

Cerano-Paredes J, J Villanueva-Díaz, P. Z. Fulé. 2010. Reconstrucción de incendios y su relación con el clima para la Reserva Cerro El Mohinora, Chihuahua. Revista Mexicana de Ciencias Forestales 1(1):63-74.

Cerano-Paredes J, J Méndez-González, A Amaro-Sánchez, J Villanueva-Díaz, R Cervantes-Martínez, EA Rubio-Camacho. 2013. Reconstrucción de precipitación invierno-primavera con anillos anuales de Pinus douglasiana en la Reserva de la Biosfera Sierra de Manantlán, Jalisco. Revista Chapingo Serie Ciencias Forestales y del Ambiente 19(3): 413-423. 
DOI: 10.5154/r.rchscfa.2013.02.007

Dieterich JH, TW Swetnam. 1984. Dendrochronology of a firescarred ponderosa pine. Forest Science 30(1): 238-247.

Englehart PJ, AV Douglas. 2001. The Role of Eastern North Pacific Tropical Storms in the Rainfall Climatology of Western Mexico. International Journal of Climatology 21: 13571370. DOI: 10.1002/joc.637

Figueroa-Rangel BL, KJ Willis, M Olvera-Vargas. 2008. 4200 years of pine dominated forest dynamics in the uplands of west-central Mexico: a human or natural legacy? Ecology 89(7): 1893-1907.

Fulé PZ, WW Covington. 1999. Fire regime changes in La Michilía Biosphere Reserve, Durango, Mexico. Conservation Biology 13(3): 640-652.

Fulé PZ, J Villanueva-Díaz, M Ramos-Gómez. 2005. Fire regime in a conservation reserve, Chihuahua, Mexico. Canadian Journal of Forest Research 35:320-330. DOI: 10.1139/ X04-173

Fulé PZ, M Ramos-Gómez, C Cortés-Montaño, AM Miller. 2011. Fire regime in a Mexican forest under indigenous resource management. Ecological Applications 21(3):764-775. DOI: 10.1890/10-0523.1

Fulé PZ, LL Yocom, C Cortés-Montaño, DA Falk, J CeranoParedes, J Villanueva-Díaz. 2012. Testing a pyroclimatic hypothesis on the Mexico-United States border. Ecology 93(8):1830-1840. DOI: 10.1890/11-1991.1

Grissino-Mayer HD, CH Baisan, TW Swetnam. 1994. Fire history and age structure analyses in the mixed conifer and spruce-fir forests of Mount Graham. Final report. Mount Graham Red Squirrel Study Committee. Phoenix, AZ, USA. US Fish and Wildlife Service. 73 p.

Grissino-Mayer HD, TW Swetnam. 2000. Century-scale climate forcing of fire regimes in the American Southwest. Holocene 10(2):213-220. DOI: 10.1191/095968300668451235

Grissino-Mayer HD. 2001. FHX2 - software for analyzing temporal and spatial patterns in fire regimes from tree rings. Tree-Ring Research 57(1):115-124.

Grissino-Mayer HD. 2010. Wildfire hazard and the role of treering research. In Stoffel $\mathrm{M}$ ed. Tree rings and natural hazards: A state-of-the-art. Berlin, Alemania. Springer-Verlag. p. 323-328.

Heinlein AH, MM Moore, PZ Fulé, WW Covington. 2005. Fire history and stand structure of two ponderosa pine-mixed conifer sites: San Francisco Peaks, Arizona, USA. International Journal of Wildland Fire 14(3): 307-320. DOI: 10.1071/WF04060-AU.

Heyerdahl EK, E Alvarado. 2003. Influence of climate and land use on historical surface fires in pine-oak forests, Sierra Madre Occidental, Mexico. In Veblen TT, WL Baker, G Montenegro, TW Swetnam eds. Fire and climatic change in temperate ecosystems of the Western Americas. New York, USA. Springer-Verlag. p. 196-217.

Holmes RL. 1983. Computer-assisted quality control in tree ring dating and measurement. Tree-Ring Bulletin 43: 69-75.

INE (Instituto Nacional de Ecología, MX). 2000. Programa de manejo de la reserva de la Biosfera Sierra de Manantlán. México D.F., México. INE-SEMARNAT. 204 p.

Jardel EJ. 1992. Estrategia para la conservación de la Reserva de la Biosfera Sierra de Manantlán. Guadalajara, México. Universidad de Guadalajara. 316 p.

Jardel EJ. 1998. Efectos ecológicos y sociales de la explotación maderera de los bosques de la Sierra de Manantlán. In Ávila R, JP Emphoux, LG Gastélum, S Ramírez, O Schöndube, F Valdez eds. El Occidente de México: arqueología, historia y medio ambiente. Perspectivas regionales. Actas del IV Coloquio Internacional de Occidentalistas. Universidad de Guadalajara / Instituto Francés de Investigación Científica para el Desarrollo en Cooperación (ORSTOM). Guadalajara, México. p. 231-251.

Jardel EJ, VR Ramírez, NF Castillo, RS García, OE BalcázarMedina, JC Chacón-Mathieu, JE Morfín. 2006. Manejo del Fuego y Restauración de Bosques en la Reserva de la Biosfera Sierra de Manantlán, México. In Flores-Garnica JG, DA Rodríguez-Trejo eds. Incendios Forestales. México y Madrid. Mundi-Prensa-CONAFOR. p. 216-242.

Jardel EJ. 2008. Sucesión ecológica y restauración de bosques subtropicales de montaña en la Estación Científica Las Joyas, México. In González-Espinosa M, JM Rey-Benayas, N Ramírez-Marcial, eds. Restauración de bosques en América Latina. México D.F., México. Fundación Internacional para la Restauración de Ecosistemas (FIRE) y Editorial MundiPrensa México. p. 77-97.

Keeley JE. 2012. Ecology and evolution of pine life histories. Annals of Forest Science 69(4): 445-453. DOI: 10.1007/ s13595-012-0201-8

Llamas CP. 2013. Régimen histórico de incendios forestales en bosques de coníferas del sur occidente de México. Tesis de Maestría en Ciencias en Manejo de Recursos Naturales. Autlán, México. Departamento de Ecología y Recursos Naturales, Universidad de Guadalajara. 80 p.

NOAA (National Oceanic and Atmospheric Administration, US). 2014. Cold and warm episodes by season. Consultado 21 mar. 2014. Disponible en http://www.cpc.ncep.noaa.gov/ products/analysis_monitoring/ensostuff/ensoyears.shtml

Poulos HM, J Villanueva-Díaz, J Cerano-Paredes, AE Camp, RG Gatewood. 2013. Human influences on fire regimes and forest structure in the Chihuahuan Desert Borderlands. Forest Ecology and Management 298: 1-11. DOI: 10.1016/j.foreco.2013.02.014

Rodríguez-Trejo DA, SJ Pyne. 1999. Mexican fires of 1998. International Forest Fire News 20: 61-63.

Rodríguez-Trejo DA, PZ Fulé. 2003. Fire ecology of Mexican pines and a fire management proposal. International Journal of Wildland Fire 12:23-37. DOI: 10.1071/WF13214.

Rubio EA. 2006. Frecuencia de incendios forestales en bosques de Pinus douglasiana del ejido Ahuacapán, Jalisco. Tesis de Licenciatura. Autlán, México. Departamento de Ecología y Recursos Naturales, Universidad de Guadalajara. 60 p.

Stephens SL, CN Skinner, SJ Gill. 2003. Dendrochronologybased fire history of Jeffrey pine-mixed conifer forests in the Sierra San Pedro Martir, Mexico. Canadian Journal of Forest Research 33:1090-1101. DOI: 10.1139/X03-031

Stokes MA, TL Smiley. 1968. An introduction to tree-ring dating. Chicago, USA. University of Chicago Press. 73 p.

Swetnam TW. 1993. Fire history and climate change in giant sequoia groves. Science 262: 885-889.

Swetnam TW, CH Baisan, JM Kaib. 2001. Forest fire histories in the sky islands of La Frontera. In Webster GL, CJ Bahre eds. Changing Plant Life of La Frontera: Observations on Vegetation in the United States/Mexico Borderlands. Albuquerque, USA. University of New Mexico Press. p. 95-119.

Swetnam TW, CH Baisan. 2003. Tree-ring reconstructions of fire 
and climate history in the Sierra Nevada and southwestern United States. In Veblen TT, WL Baker, G Montenegro, TW Swetnam eds. Fire and climatic change in temperate ecosystems of the western Americas. New York, USA. Springer-Verlag. p. 158-195.

Trenberth KE. 1997. The definition of El Niño. Bulletin of the American Meteorological Society 78(12): 2771-2777. DOI: 10.1175/1520-0477

Vélica-Zúñiga G. 2013. Vegetación y patrones geoecológicos de la Reserva de la Biosfera Sierra de Manantlán. Tesis de Maestría en Ciencias en Manejo de Recursos Naturales.
Autlán, México. Departamento de Ecología y Recursos Naturales, Universidad de Guadalajara. 101 p.

Yocom LL, PZ Fulé, PM Brown, J Cerano-Paredes, J VillanuevaDíaz, DA Falk, E Cornejo-Oviedo. 2010. El Niño Southern Oscillation effect on a fire regime in northeastern Mexico has changed over time. Ecology 91(6):1660-1671. DOI: 10.1890/09-0845.1

Yocom LL, PZ Fulé. 2012. Human and climate influences on frequent fire in a high-elevation tropical forest. Journal of Applied Ecology 49(6): 1356-1364. DOI: 10.1111/j.13652664.2012.02216.x

Recibido: 20.08.14

Aceptado: 18.12 .14 